\title{
In vitro SIV replication kinetics correlate with vaccine induced cellular immune responses and predict post-challenge outcome in immunized rhesus macaques

\author{
Washingtone Ochieng*, Christiane Stahl-Hennig, You-Suk Suh, \\ Sieghart Sopper, Gerhard Hunsmann and Ulrike Sauermann
}

Address: Department of Virology and Immunology, German Primate Centre, Goettingen, Germany, Kellnerweg 4, 37077

Email: Washingtone Ochieng* - wochieng@dpz.gwdg.de

* Corresponding author

from 2006 International Meeting of The Institute of Human Virology

Baltimore, USA. 17-21 November, 2006

Published: 21 December 2006

Retrovirology 2006, 3(SuppI I):P46 doi:I0.I I86/I742-4690-3-SI-P46

(C) 2006 Ochieng et al; licensee BioMed Central Ltd.

Strong and sustained immune response is central in AIDS vaccine research. Here, we use an in vitro model to describe correlation kinetics of virus replication and T-cell responses. Eighteen rhesus monkeys were recruited into Group- 1 (controls) and groups 2 and 3 which were DNAprimed followed by adenovirus-vaccine boost via different routes. All animals were challenged with SIVmac239 after 44 weeks. During immunization, ex-vivo interferon gamma (IFN- $\gamma$ ) responses and in vitro SIV suppressor activities (VSA) in cell-culture were determined respectively using ELISPOT and a non-cytotoxic antiviral activity assay. Virus replication efficiency in vitro (VVR) and after challenge was measured using real-time PCR. At baseline, VVR was comparable in all groups and remained constant in controls. However, VVR declined significantly $(\mathrm{p}=$ 0.001 ) in vaccines, correlated with increased IFN- $\gamma$ responses $(p=0.019)$ and VSA $(p=0.05)$. Peak viremia post-challenge was significantly lowered in vaccinnes $(\mathrm{p}=$ $0.006)$ and correlated with in vitro kinetics for control animals. Acute-phase set point correlated with VSA ( $\mathrm{p}=$ 0.001 ) but not IFN- $\gamma$ levels. Our in vitro model predicts post-challenge outcome and implicates multifactorial cellular immune factors in controlling viral replication. Optimizing these immune components in candidate vaccine designs may improve potency and outcome. 